\title{
Nuevo cuestionario para evaluar la autoeficacia hacia la actividad física en niños
}

\author{
Ángeles Aedo ${ }^{1}$ y Héctor Ávila ${ }^{2}$
}

Forma de citar

Aedo A, Ávila H. Nuevo cuestionario para evaluar la autoeficacia hacia la actividad física en niños. Rev Panam Salud Publica. 2009;26(4):324-9.

RESUMEN Objetivos. Desarrollar un cuestionario para evaluar autoeficacia hacia la actividad física en niños de edad escolar, así como medir su validez de constructo, confiabilidad test-retest y consistencia interna.

Método. Se utilizó un multimétodo en cuatro etapas: 1) Investigación bibliográfica y consecutivamente un estudio exploratorio. Construcción de los reactivos del cuestionario utilizando una escala dicotómica de 14 ítems. 2) Evaluación de la validez de contenido a través de un panel de expertos. 3) Aplicación de la primera versión del cuestionario final a una muestra de 900 niños de edad escolar de la ciudad de México. 4) Determinación de la validez de constructo, la confiabilidad test-retest y la consistencia interna (Alfa de Cronbach).

Resultados. Se obtuvieron tres factores que explican el 64,15\% de la varianza: búsqueda de alternativas positivas hacia la actividad física, capacidad para enfrentar posibles barreras para llevarla a cabo y expectativas de habilidad o competencia. Se validó el modelo por medio de la bondad del ajuste, obteniéndose un $65 \%$ de residuos inferiores a 0,05 , resultado indicativo de que el modelo factorial estimado se ajusta a los datos. La consistencia alfa de Cronbach fue de 0,733 , la confiabilidad test-retest fue de 0,867 .

Conclusiones. Se desarrolló una escala con validez y confiabilidad adecuadas. Estos resultados permiten utilizarla como un buen indicador de la autoeficacia hacia la actividad física en niños de edad escolar, lo cual es importante en el desarrollo de programas cuyo objetivo sea fomentar dicha conducta en este grupo de edad.

Palabras clave Actividad física; autoeficacia; confiabilidad y validez; cuestionario; ejercicio físico; niño; salud del niño; México.

Realizar actividad física como parte del estilo de vida tiene muchas ventajas, incluyendo un riesgo menor de sufrir en-

\footnotetext{
Facultad de Medicina, Universidad Autónoma de México, México DF, México. La correspondencia debe dirigirse a Ángeles Aedo, Universidad Autónoma de México, Facultad de Medicina, Av. Universidad 3000, Circuito Escolar, Ciudad Universitaria, México 04510, México. Correo electrónico: aedoaa@gmail.com

2 Departamento de Investigación en Epidemiología, Instituto Nacional de Pediatría, Secretaría de Salud de México, México DF, México.
}

fermedad coronaria, hipertensión arterial, cáncer de colon, obesidad y osteoporosis. Más aún, el ejercicio físico reduce el estrés, la ansiedad y los síntomas depresivos, al tiempo que incrementa el bienestar emocional, el nivel percibido de energía, la autoestima y la satisfacción personal (1-5). Contrariamente, el sedentarismo aumenta el riesgo de adquirir enfermedades crónicas no transmisibles. En el caso de los niños y los adolescentes, la falta de ejer- cicio tiene consecuencias todavía más graves porque eleva el riesgo de adquirir enfermedades propias de la vida adulta, como diabetes y enfermedades coronarias $(6,7)$.

A pesar de los bien documentados beneficios de la actividad física, en varios países se ha observado una disminución preocupante de esta conducta en la población, un problema que lamentablemente alcanza también a los niños, quienes en su mayoría no cubren los niveles 
mínimos de ejercicio recomendados para proteger su salud (8).

El proceso de modificación de conducta que supone la ruptura del sedentarismo debe iniciarse en edades tempranas, cuando es más fácil desarrollar hábitos saludables que hacerlo una vez que se llega a la edad adulta. En consecuencia, dado que la infancia es el período evolutivo ideal para adoptar estilos de vida sanos, los programas dirigidos a promover comportamientos saludables en la familia, incluida la actividad física, deben empezar precisamente en esta etapa (9).

Existen diferentes modelos teóricos que intentan explicar la conducta hacia la actividad física, entre ellos la Teoría de la Conducta Planificada y la Teoría Social Cognitiva - especialmente la autoeficacia- dos modelos que han demostrado ser capaces de predecir adecuadamente la actividad física tanto en niños como en adultos $(10,11)$. En realidad se podría decir que hay un traslape entre la autoeficacia de la Teoría Social Cognitiva y la percepción de control sobre la conducta de la Teoría de la Conducta Planificada, es decir, que representan un mismo constructo.

La autoeficacia es la convicción de que se tendrá éxito al llevar a cabo la conducta en cuestión, la confianza en los resultados que tiene una persona al decidir cambiar o mantener cierta acción o conducta (12). Tal convicción influye en cómo la persona encara los desafíos y las metas que se plantea, el grado de esfuerzo que invierte en lograrlas, los resultados que espera alcanzar y la magnitud de su perseverancia frente a los obstáculos (13). Este constructo puede medirse a través de sus dimensiones: la búsqueda de alternativas positivas hacia la conducta, la capacidad para enfrentar posibles barreras para llevarla a cabo y las expectativas de habilidad o competencia.

A continuación se mencionan algunos estudios que han investigado la relación entre la autoeficacia y la actividad física, así como sus principales hallazgos:

- Ferguson et al. (14) realizaron en 1989 un estudio en 603 adolescentes de Iowa, Estados Unidos, donde concluyeron que el desarrollo temprano de actitudes positivas hacia esta conducta tiene un papel importante sobre la inclinación personal a tener un estilo de vida activo.
- En 1994 Biddle y Goudas estudiaron en el Reino Unido a 147 niños de 13 y 14 años de edad. La percepción del control de esta conducta correlacionó significativamente con la misma (15). En un estudio longitudinal de 1994 realizado en sitios de trabajo de Rhode Island, Estados Unidos, Marcus et al. (16) concluyeron que la autoeficacia aparece como un importante indicador de la conducta hacia el ejercicio.

- En 1995 Kimiecik et al. estudiaron una muestra de 81 niños de 11 a 15 años de edad en Ohio, Estados Unidos, encontrando una correlación significativa entre la autoeficacia y la actividad física de estos niños (17).

- En 2005 Armitage realizó en el Reino Unido un estudio longitudinal en 94 adultos, en el cual la percepción de control de la conducta predijo significativamente la actividad física de la muestra (18).

- En 2005 Sniehotta et al. observaron que en una muestra de 307 pacientes cardíacos alemanes, la autoeficacia funcionó como mediadora entre las intenciones iniciales de realizar ejercicio y la actividad física realizada posteriormente (19).

- En un estudio llevado a cabo en 2006 por Jeffrey et al. en una muestra de niños méxico-americanos de 9 a 12 años de edad residentes en Detroit, Estados Unidos, se concluyó que tanto las actitudes como la norma subjetiva y la percepción del control predijeron la actitud hacia la actividad física (20).

Aunque la autoeficacia percibida se concibe como uno de los principales determinantes de las intenciones de hacer ejercicio y de seguir haciéndolo por largos períodos de tiempo, son muy pocos los cuestionarios de autoeficacia elaborados específicamente para actividad física y aún menos para medir este constructo en la población infantil (21). El objetivo principal del presente trabajo fue justamente desarrollar un instrumento para medir la autoeficacia en su relación con la actividad física (cuestionario) en niños de edad escolar, así como su validez de constructo, confiabilidad test-retest $\mathrm{y}$ consistencia interna.

\section{MATERIALES Y MÉTODOS}

El diseño, la construcción y la validación del cuestionario se realizó utilizando procesos cualitativos y cuantita- tivos, que se llevaron a cabo en cuatro etapas:

1) Desarrollo del borrador inicial. El primer paso fue identificar los constructos objeto de estudio (el dominio del contenido) por medio de una investigación bibliográfica. Luego se efectuó un estudio exploratorio que consistió en una entrevista semi-estructurada a 40 madres y padres de niños de 7 a 10 años y 11 meses de edad, en la que se les preguntó sobre sus creencias con respecto a la salud y la actividad física, y su percepción acerca de barreras y facilidades para realizar esta actividad en el vecindario en el que viven. El propósito del estudio fue enriquecer el diseño del cuestionario, tomando en cuenta el panorama de quienes son los principales responsables del desarrollo de hábitos a esta edad, dentro de su contexto social y cultural. Los resultados, junto con los datos teóricos encontrados en la revisión bibliográfica y el análisis de estudios anteriores, conformaron la plataforma sobre la que se construyó el cuestionario en el que se utilizó una escala dicotómica de 14 reactivos, que abarcaron tres dimensiones de la autoeficacia: la búsqueda de alternativas positivas, la capacidad para enfrentar posibles barreras y las expectativas de habilidad o competencia. Se asignaron los valores 1 y 2 a la escala dicotómica (que significan sí y no, respectivamente).

2) Evaluación de validez de contenido. Se seleccionó un panel de expertos (siete jueces) con base en los siguientes requisitos: que fueran profesionales de la salud; que tuvieran experiencia y reconocida competencia en educación para la salud, uso (desarrollo, evaluación y validación) de instrumentos psicométricos y actividad física en niños; y que aceptaran evaluar la primera versión del instrumento de recolección de datos. Estos expertos revisaron cualitativa y cuantitativamente el cuestionario, con el fin de mejorar la calidad de los reactivos y eliminar los posibles errores provenientes de la estructura del instrumento, además de ser parte importante de la validación de constructo.

En la revisión cualitativa se determinó si las instrucciones del cuestionario eran apropiadas, concisas y completas, y si los reactivos fueron claros. La evaluación cuantitativa consistió en establecer un índice numérico para la validez de contenido del cuestionario, utilizándose la fórmula y metodología propuesta por 
Lawshe y perfeccionada por Veneziano y Hooper (22), que cuantifica el grado de consenso del panel de expertos para cada reactivo del cuestionario; cada miembro del panel evaluó cada reactivo del cuestionario y determinó si era representativo del concepto que el instrumento pretendía medir. Los miembros decidieron si cada reactivo era: "esencial", "útil pero no esencial" o "no necesario" para lograr el objetivo del cuestionario. Las respuestas de los panelistas se agruparon y se contabilizó el número de panelistas que calificaron el reactivo como "esencial". Se procedió a obtener el índice de validez de contenido (IVC) para cada reactivo:

$$
I V C=\frac{n_{e}-N / 2}{N / 2}
$$

donde $n_{e}$ es el número de panelistas que indicaron el reactivo como "esencial" y $N$ es el total de panelistas.

El IVC es una medición estadística que permite determinar si el reactivo se elimina o se retiene en el cuestionario. Cada reactivo que resultó con un valor menor a 0,70 se eliminó; en el cuadro 1 se muestran los valores del IVC para los 14 reactivos. De los 14 reactivos que componían inicialmente el cuestionario se decidió desechar tres debido a su bajo IVC, y se incluyó un reactivo más por recomendación de los expertos, obteniéndose así la primera versión del cuestionario compuesta por 12 reactivos.

\section{CUADRO 1. Índice de validez de conte- nido (IVC) a de los reactivos del cuestio- nario de autoeficacia hacia la actividad física, México, 2007}

\begin{tabular}{cr}
\hline Reactivo & IVC \\
\hline 1 & 0,99 \\
2 & 0,14 \\
3 & 0,71 \\
4 & 0,71 \\
5 & 0,71 \\
6 & 0,14 \\
7 & $-0,14$ \\
8 & 0,71 \\
9 & 0,99 \\
10 & 0,99 \\
11 & 0,99 \\
12 & 0,99 \\
13 & 0,99 \\
14 & 0,71 \\
\hline
\end{tabular}

a EI IVC representa el grado de consenso del panel de expertos con respecto a si el reactivo es esencial o no para lograr el objetivo del cuestionario. El punto de corte para eliminar el reactivo es 0,70 .
3) Estudio de campo. La primera versión del cuestionario final se aplicó a una muestra de 900 niños de 7 a 10 años y 11 meses de edad residentes en la ciudad de México. Se usó un diseño de muestral polietápico, utilizando como marco muestral las escuelas primarias de dos delegaciones: Magdalena Contreras y Tlalpan, seleccionadas por tener AGEB's (áreas geoestadísticas básicas) con los cinco diferentes grados de marginación utilizados por el Consejo Nacional de Población (CONAPO) y por el Instituto Nacional de Estadística, Geografía e Informática (INEGI) como criterio de grado de pobreza y marginación en México $(23,24)$. Como unidad última de muestreo se consideró a los niños de 7 a 10 años y 11 meses de edad que asisten a las escuelas primarias incluidas en el estudio.

Para esta etapa se utilizó un muestreo estratificado por grado escolar y sexo, con objeto de tener representatividad suficiente de niños de segundo a quinto grado de primaria, incluyendo a los que al momento del estudio tuvieran edades de 7 a 10 años y 11 meses. Del total de los niños estudiados, 685 (76\%) pertenecían a escuelas públicas y $215(24 \%)$ a escuelas privadas -462 (52\%) varones y 438 (48\%) niñas- (cuadro 2). Se guardó la proporción de 3:1 que existe entre el número de niños que asisten respectivamente a escuelas públicas y privadas en la ciudad de México.

4) Evaluación de validez de constructo, confiabilidad test-retest y consistencia interna. Para evaluar la validez de constructo se realizó inicialmente un análisis estadístico de los reactivos. Se calcularon la media y la desviación estándar (DE) de cada reactivo, considerando los más adecuados aquellos que presentan una DE cercana a 0,5 . Para comprobar si las características del cuestionario eran adecuadas para rea-

lizar el análisis factorial se utilizó la medida de adecuación de la muestra índice de Kaiser-Meyer-Olkin (KMO), que es una medida que toma valores de entre cero y uno, y se utiliza para comparar las magnitudes de los coeficientes de correlación observados con las magnitudes de los coeficientes de correlación parcial. Kaiser, Meyer y Olkin aconsejan que si el KMO es $\geq 0,75$, la idea de realizar un análisis factorial es buena, si el KMO siendo $<0,75$, es $\geq 0,5$, la idea es aceptable y si el KMO es $<0,5$, inaceptable (25).

Posteriormente se realizó un análisis factorial de componentes principales con rotación VARIMAX, el cual permitió agrupar los reactivos relacionados que se enlazaron como conceptos unificados (variables). Se consideró que el reactivo pertenecía a un factor si su correlación fue igual o mayor a 0,35 (26). El modelo factorial se validó a través de la bondad de ajuste: dado que una suposición básica subyacente al análisis factorial es que la correlación observada entre las variables puede atribuirse a factores comunes, la bondad del ajuste se determina estudiando las diferencias entre las correlaciones observadas (como se dan en la matriz de correlación de entrada) y las correlaciones reproducidas (como se estiman a partir de la matriz factorial). Estas diferencias se conocen como residuos. Si el modelo factorial es adecuado, entonces estos residuos deben ser iguales o menores a 0,05 , lo que significa que el modelo factorial estimado se ajusta a los datos (27).

Para evaluar la confiabilidad del instrumento se utilizaron dos métodos:

1) Confiabilidad por consistencia interna (Alfa de Cronbach), cuyo resultado da una escala de valores de 0 a 1 ; valores mayores de 0,70 indican una consistencia

CUADRO 2. Distribución de la muestra de 900 niños según tipo de escuela, grado escolar y sexo, México, 2007

\begin{tabular}{|c|c|c|c|c|c|c|c|c|}
\hline \multirow[b]{3}{*}{ Grado } & \multicolumn{4}{|c|}{ Escuela pública } & \multicolumn{4}{|c|}{ Escuela privada } \\
\hline & \multicolumn{2}{|c|}{ Varones } & \multicolumn{2}{|c|}{ Niñas } & \multicolumn{2}{|c|}{ Varones } & \multicolumn{2}{|c|}{ Niñas } \\
\hline & No. & $\%$ & No. & $\%$ & No. & $\%$ & No. & $\%$ \\
\hline $2^{\circ}$ & 89 & 26,2 & 84 & 24,3 & 26 & 21,1 & 27 & 29,3 \\
\hline $3^{\circ}$ & 81 & 23,8 & 85 & 24,6 & 32 & 26 & 20 & 21,7 \\
\hline $4^{\circ}$ & 87 & 25,7 & 92 & 26,6 & 32 & 26 & 17 & 18,5 \\
\hline $5^{\circ}$ & 82 & 24,2 & 85 & 24,6 & 33 & 26,8 & 28 & 30,4 \\
\hline Total & 339 & 100 & 346 & 100 & 123 & 100 & 92 & 100 \\
\hline
\end{tabular}

Nota: se incluyeron en el estudio todos los niños que al momento de la aplicación del cuestionario tenían desde 7 hasta 10 años y 11 meses de edad. 
interna aceptable y sugieren que el instrumento puede aplicarse (28).

2) Confiabilidad test-retest, que consistió en administrar el cuestionario dos veces (con dos meses de diferencia) a los mismos sujetos. Se correlacionaron ambas aplicaciones utilizando el coeficiente de correlación de Pearson, cuyos valores mayores a 0,75 se consideran aceptables (29).

\section{RESULTADOS}

En el cuadro 3 se puede observar la media y las DE de los reactivos del cuestionario. Las DE se acercan a 0,5 , por lo que puede considerarse que los reactivos presentan variabilidad adecuada en la muestra. La consistencia interna alfa de Cronbach de esta escala fue de 0,733 $(0,735$ estandarizado). La segunda aplicación del cuestionario se realizó a 860 de los 900 niños en quienes se aplicó la primera vez, y el coeficiente de confiabilidad test-retest resultante fue de 0,867. El índice KMO fue de 0,639; aunque no muy alto, es aceptable para realizar el análisis factorial.

En el cuadro 4 se muestran los resultados del análisis de componentes principales con rotación VARIMAX, del cual se obtuvieron tres factores que explican el $64,15 \%$ de la varianza. El primer factor representa la búsqueda de alternativas positivas hacia la actividad física, y está compuesto por tres componentes que explican el 31,4\% de la varianza. El segundo factor fue la capacidad para enfrentar posibles barreras para llevar a cabo la actividad física, que explica casi un $20 \%$ de la varianza y consta de cinco componentes. El tercer factor representa las expectativas de habilidad o competencia, explica el $13 \%$ de la varianza y está compuesto por tres componentes. El reactivo 3 ("Yo puedo hacer ejercicio o deporte después de la escuela, aunque mis amigos quieran que haga alguna otra cosa"), cuya correlación es menor a 0,35 en todos los casos, no pertenece a ningún factor y se optó por eliminarlo.

El modelo factorial resultante se considera adecuado ya que, después de validarlo a través de la bondad de ajuste, se obtuvo un $65 \%$ de residuos inferiores a 0,05 , resultado indicativo de que se ajusta a los datos.

\section{DISCUSIÓN}

Se diseñó y validó un cuestionario para evaluar la autoeficacia hacia la acti- vidad física en niños de edad escolar. Aunque múltiples estudios han utilizado la autoeficacia para tratar de explicar la actividad física, esta es la primera vez que en México se desarrolla un instrumento de esta naturaleza para este grupo de población $(30,31)$. La confiabilidad test-retest obtenida demuestra que la escala desarrollada tiene un alto grado de congruencia y utilizando el coeficiente alfa de Cronbach, se puede afirmar que tiene una adecuada consistencia interna entre sus reactivos.

El instrumento aquí descrito ha mostrado características psicométricas adecuadas y una importante consistencia con escalas similares a las utilizadas anteriormente por otros autores. Los valores de consistencia interna alfa de Cronbach son mayores a los obtenidos en una escala similar desarrollada por Saunders et al. (32) para preadolescentes afroamericanos (alfa $=0,62)$ y a los obtenidos por Biddle y Goudas (15) en un grupo de niños de 13 y 14 años (alfa $=0,51$ ); y semejantes a los obtenidos en la escala desarrollada por Godin y Shepard (33) para adolescentes

de $7^{\circ}$ a $9^{\circ}$ grado escolar en California y por Trost et al. (34), en niños de $6^{\circ}$ grado (alfa $=0,72$ ). Hay otras escalas diseñadas para otros grupos de edad cuya consistencia interna es mayor, por ejemplo la de Kroll et al. (35), validada en 368 adultos

CUADRO 3. Media y desviación estándar (DE) de los reactivos del cuestionario de autoeficacia, México, 2007

\begin{tabular}{rrr}
\hline Reactivo & Media & DE \\
\hline 1 & 1,218 & 0,413 \\
2 & 1,433 & 0,496 \\
3 & 1,285 & 0,452 \\
4 & 1,249 & 0,433 \\
5 & 1,407 & 0,492 \\
6 & 1,563 & 0,496 \\
7 & 1,517 & 0,500 \\
8 & 1,219 & 0,414 \\
9 & 1,233 & 0,423 \\
10 & 1,482 & 0,500 \\
11 & 1,249 & 0,433 \\
12 & 1,250 & 0,433 \\
\hline
\end{tabular}

Nota: No. de la muestra $=900$. Los valores medios se encuentran entre 1 y 2 debido a que la escala es dicotómica. Todos los reactivos presentaron una variabilidad cercana a 0,5 , lo que se considera adecuado en este tipo de escalas.

CUADRO 4. Análisis factorial de componentes principales con rotación VARIMAX para la escala de autoeficacia hacia la actividad física, México, 2007

\begin{tabular}{|c|c|c|c|c|}
\hline \multirow[b]{2}{*}{ Reactivo } & \multirow[b]{2}{*}{ Componente } & \multicolumn{3}{|c|}{ Factor } \\
\hline & & $\begin{array}{c}\text { I } \\
\text { Alternativas } \\
\text { positivas }\end{array}$ & $\begin{array}{c}\text { II } \\
\text { Superación } \\
\text { de barreras }\end{array}$ & $\begin{array}{c}\text { III } \\
\text { Expectativas } \\
\text { de habilidad }\end{array}$ \\
\hline & Yo creo que puedo: & & & \\
\hline 1 & Hacer algo de actividad física después de la escuela & & & \\
\hline & la mayoría de los días entre semana & 0,431 & 0,098 & 0,229 \\
\hline 2 & Hacer actividad física después de la escuela aunque & & & \\
\hline & también vea TV o juegue videojuegos & $-0,083$ & 0,499 & 0,118 \\
\hline 3 & Hacer ejercicio o deporte después de la escuela & & & \\
\hline & aunque mis amigos quieran que haga alguna otra cosa & 0,192 & 0,120 & 0,286 \\
\hline 4 & Correr al menos 8 minutos sin parar & 0,229 & $-0,042$ & 0,396 \\
\hline 5 & Hacer actividad física aunque haga calor o frío afuera & 0,051 & 0,586 & 0,035 \\
\hline 6 & Hacer ejercicio aunque me sienta cansado & 0,132 & 0,424 & 0,026 \\
\hline 7 & Hacer actividad física aunque tenga mucha tarea & $-0,015$ & 0,678 & 0,077 \\
\hline 8 & Hacer actividad física aunque me quede en casa & 0,812 & 0,080 & $-0,019$ \\
\hline 9 & Hacer ejercicio o algún deporte aunque mis amigos & & & \\
\hline & crean lo contrario & 0,046 & 0,145 & 0,833 \\
\hline 10 & Hacer actividad física aunque tenga otras clases en & & & \\
\hline & las tardes & 0,164 & 0,699 & 0,126 \\
\hline & Yo creo que: & & & \\
\hline 11 & Tengo la habilidad necesaria para jugar el deporte que & & & \\
\hline & quiera o para hacer ejercicio & 0,042 & 0,078 & 0,868 \\
\hline 12 & Alguno de mis padres (o adulto que me cuida) puede & & & \\
\hline & llevarme a practicar deporte o hacer ejercicio en la tarde & 0,847 & 0,073 & $-0,078$ \\
\hline & Eigenvalue (autovalores) ${ }^{\mathrm{a}}$ & 3,763 & 2,548 & 1,566 \\
\hline & $\%$ varianzab & 31,358 & 19,755 & 13,051 \\
\hline $\begin{array}{l}\text { Nota: núm } \\
\text { zación Va } \\
\text { a Represen } \\
\text { decidir el } \\
\text { formación }\end{array}$ & $\begin{array}{l}\text { ero de la muestra }=860 \text {. Método de extracción: análisis de compone } \\
\text { rimax con Kaiser. } \\
\text { tan la suma de los cuadrados de los pesos de las columnas de la m } \\
\text { número de factores presentes. Sólo se incluyen aquellos que son m } \\
\text { explicada por el modelo. }\end{array}$ & $\begin{array}{l}\text { entes principale } \\
\text { matriz factorial, } \\
\text { mayores a } 1 . \mathrm{D}\end{array}$ & $\begin{array}{l}\text { es. Método de } \\
\text {, y se utilizan } \\
\text { Jan cuenta de }\end{array}$ & $\begin{array}{l}\text { mo criterio para } \\
\text { cantidad de in- }\end{array}$ \\
\hline
\end{tabular}


de Estados Unidos (alfa = 0,92). Otra escala de este tipo es la Autoeficacia para el Entrenamiento Físico, desarrollada por Bandura (36) para población adulta, que ha sido traducida a diferentes idiomas, y en su versión en español obtuvo un alto puntaje de confiabilidad (alfa $=0,93$ ), mientras que en inglés presentó una consistencia interna alfa de 0,92 (37).

La Escala de Autoeficacia General es un instrumento psicométrico de 10 reactivos diseñado para determinar el grado en que las personas creen que pueden hacer frente con éxito a una variedad de posibles dificultades en la vida. Fue desarrollada inicialmente en alemán por Jerusalén y Schwarzer en 1981 y traducida a 30 idiomas (38). En los distintos estudios realizados, con muestras de varias nacionalidades, esta escala ha mostrado una consistencia interna considerable (entre 0,79 y 0,93). En la versión española se obtuvo una consistencia interna alfa de Cronbach de 0,87 y una correlación entre dos mitades de 0,88. Estos resultados son ligeramente mayores a los obtenidos en la escala del presente estudio.

El haber realizado un estudio multimétodo-multietápico, tomando en cuenta para la construcción del cuestionario los resultados obtenidos en el estudio exploratorio y en el estudio de validez de contenido, aumentó la probabilidad de obtener una adecuada validez de constructo, por lo que se recomienda seguir estas etapas para el desarrollo y validación de este tipo de instrumentos.

Después de este proceso, puede afirmarse que la estructura empírica de esta escala se corresponde con la estructura teórica planteada (cuadro 4). El presente trabajo corroboró la estructura factorial de la autoeficacia, lo que pone de manifiesto la naturaleza multifactorial de este constructo. Los factores obtenidos corresponden a una estructura trifactorial, en la que destacan las siguientes dimensiones que pueden ser utilizadas como variables:

Factor I. La búsqueda de alternativas positivas hacia la actividad física, que denota la capacidad que poseen los niños de explorar posibles alternativas para la realización de la actividad física y explica un porcentaje elevado de la varianza.

Factor II. La capacidad de enfrentar posibles barreras para realizar la actividad física, que implica la búsqueda de soluciones a los obstáculos que encuentren para realizar la conducta.

Factor III. Las expectativas de habilidad o competencia, que se refieren a la confianza que poseen los niños en su capacidad para realizar adecuadamente la conducta. No evalúa las destrezas o habilidades del niño, sino su propio juicio acerca de ellas.

Una limitante del presente estudio es que, debido al nivel de desarrollo cognitivo y al grado de comprensión que muestran sus participantes, se utilizó una escala dicotómica (sí o no) en lugar de las escalas de cinco puntos utilizadas comúnmente para este tipo de instrumentos. Tal restricción en la variabilidad de las respuestas puede llegar a dificultar la detección de la relación entre la autoeficacia y la actividad física. El rango de edad elegido para los participantes —de 7 a 10 años y 11 meses- obedeció a que los menores de 7 años no tienen el desarrollo cognitivo necesario para responder adecuadamente el cuestionario, y que en mayores de 11 años es más apropiado el uso de otro tipo de escalas, como por ejemplo la de Likert.

Valdría la pena refrendar los resultados obtenidos en el análisis factorial realizando estudios sobre nuevas muestras extraídas de la población objetivo o de submuestras de esta misma muestra, para poder generalizar más los resultados. Debido a que la validación del cuestionario se realizó en población urbana, se recomienda que su utilización en poblaciones rurales sea precedida por el estudio de validación correspondiente, ya que las condiciones y estilos de vida de los niños de ambas poblaciones son diferentes.

Los resultados obtenidos en el presente estudio permiten recomendar que este instrumento se comience a utilizar en la planificación y el diseño de programas para aumentar la práctica de actividad física en los niños, que realizan instituciones dedicadas al cuidado y la atención de la salud como parte de una estrategia para el combate al sobrepeso, la obesidad y las enfermedades crónicas no transmisibles.

Dado que conocer el nivel de autoeficacia de los niños en edad escolar hacia la actividad física puede tener implicaciones importantes en el desarrollo de estrategias para la promoción de esta conducta, el instrumento aquí descrito puede considerarse relevante por su capacidad demostrada de predecir en medida adecuada los patrones de comportamiento hacia dicha actividad. En conclusión, se desarrolló un cuestionario con confiabilidad y validez suficientes como para utilizar la medida de autoeficacia percibida hacia la actividad física como un buen indicador para el conocimiento de esta variable en la población infantil.

\section{REFERENCIAS}

1. Moraska A, Fleshner N. Voluntary Physical Activity Prevents Stress, Induced Behavioral Depression and Anti-KLH Antibody Suppression. Am J Physiol. 2000;281:484-9.

2. Hernández B, Gortmaker S, Laird N, Colditz G, Parra-Cabrera S, Peterson K. Validez y reproducibilidad de un cuestionario de actividad e inactividad física para escolares de la Ciudad de México. Salud Publica Mex. 2000; 42(4):315-23.

3. Pianosi P, Davis H. Determinants of Physical Fitness in Children with Asthma. Pediatrics. 2004;113(3):225-9.

4. Salmon J, Timperio A. Prevalence Trends and Environmental Influences on Child and Youth Physical Activity. Med Sport Sci. 2007;50: 183-99.
5. Sherwood N, Jeffery R. The Behavioral Determinants of Exercise: Implications for Physical Activity Interventions. Annu Rev Nutr. 2000; 20:21-44.

6. Juarbe T, Lipson J, Turok X. Physical Activity Beliefs, Behaviours and Cardiovascular Fitness of Mexican Immigrant Women. J Transcult Nurs. 2003;14(2):108-16.

7. Daniels SR, Greer FR. Committee on Nutrition. Lipid Screening and Cardiovascular Health in Childhood. Pediatrics. 2008;122(1): 198-208.

8. Corbin C, Dale D. Measurement Issues in the Assessment of Physical Activity in Children. Res Q Exerc Sport. 2000;71(2):59-71.

9. Hallal P, Wells J, Reichert F, Anselmi L, Victora C. Early Determinants of Physical Activ- ity in Adolescence: Prospective Birth Cohort Study. Br Med J. 2006;332:1002-7.

10. Courneya K, Plotnikoff R, Hotz S, Birkett N. Social Support and the Theory of Planned Behavior in the Exercise Domain. Am J Health Behav. 2000;24(4):300-8.

11. Bandura A. Social Cognitive Theory: An Agentic Perspective. Annu Rev Psychol. 2001; 52:1-26.

12. Bandura A. Self-efficacy, the Exercise of Control. New York: WH Freeman; 1998.

13. Devellis B, Devellis R. Self-efficacy and Health. En: Baum T, Revenson A, Singer J, eds. Handbook of Health Psychology. Mahwah, NJ: Lawrence Erlbaum; 2000. Pp. 235-47.

14. Ferguson K, Yesalts C, Pomrehn P, Kirkpatrick A. Attitudes, Knowledges and Beliefs as Pre- 
dictors of Exercise Intent and Behavior in Schoolchildren. J Sch Health. 1989;59(11):12-24.

15. Biddle S, Goudas M. Analysis of Children's Physical Activity and its Association with Adult Encouragement and Social Cognitive Variables. J Sch Health. 1996;66(2):75-84.

16. Marcus B, Eaton C, Rossi J, Harlow L. Selfefficacy, Decision Making and Stages of Change: An Integrative Model of Physical Exercise. J Appl Soc Psychol. 1994;24:489-508.

17. Kimiecik J, Horn T, Shurin C. Relationships Among Children's Beliefs, Perceptions of their Parents' Beliefs and their Moderate-tovigorous Physical Activity. Res Q Exerc Sport. 1996;63(3):324-36.

18. Armitage C. Can the Theory of Planned Behavior Predict the Maintenance of Physical Activity? Health Psychol. 2005;24(3):235-45.

19. Sniehotta R, Scholz U, Schwarzer R. Bridging the Intention-behavior Gap: Planning, Selfefficacy, and Action Control in the Adoption and Maintenance of Physical Exercise. Psychol Health. 2005;20(2):143-60.

20. Jeffrey J, Kimberly L, Mc Caughtry N. The Theory of Planned Behavior: Predicting Physical Activity in Mexican-american Children. J Sports Exerc Psychol. 2007;29(2):225-38.

21. Gao Z, Xiang P, Lee AM, Harrison L. Selfefficacy and Outcome Expectancy in Beginning Weight Training Class: their Relations to Students' Behavioral Intention and Actual Behavior. Res Q Exerc Sport. 2008;79(1):92-100.

22. Veneziano L, Hooper J. A Method for Quantifying Content Validity of Health-related Questionnaires. Am J Health Behav. 1997;21(1): $67-70$.
23. Instituto Nacional de Estadística, Geografía e Informática de México. XII Censo general de población y vivienda, 2000. COPLADEGDF. Hallado en: www.inegi.gob.mx. Acceso el 7 de mayo de 2006.

24. Solis P. Marginación urbana en la situación demográfica de México. México: CONAPO; 2002. P. 120.

25. Pérez Gil J, Moscoso S, Rodríguez R. Validez de constructo: el uso del análisis factorial exploratorio-confirmatorio para obtener evidencia de validez. Psicothema. 2000;12(2):442-6. Disponible en: http://www.psicothema.com/ pdf/601.pdf. Acceso el 14 de agosto de 2009.

26. Cortada N. Técnicas psicológicas de evaluación y exploración. México: Trillas; 2000. Pp. 56-88.

27. Universidad Autónoma de Madrid. Análisis factorial. Cap. 20. Hallado en: http://www. ucm.es/info/socivmyt/paginas/D_departa mento/materiales/analisis datosyMulti variable/20factor_SPSS.pdf. Acceso el $16 \mathrm{de}$ septiembre de 2009.

28. Argimon J, Jiménez J. Métodos de investigación clínica y epidemiológica. Madrid: Harcourt; 1999. Pp. 104-18, 168-72.

29. Stevens J, Cornell C, French S, Levin S, Becenti A, Gittelsohn J, et al. Development of a Questionnaire to Asses Knowledge, Attitudes and Behaviors in American Indian Children. Am J Clin Nutr. 1999;69(4):S773-81.

30. Dishman RK, Motl RW, Sallis JF, Dunn AL, Birnbaum AS, Welk GJ, et al. Self-management Strategies Mediate Self-efficacy and Physical Activity. Am J Prev Med. 2005;29(1):10-8.

31. Motl RW, Dishman RK, Saunders RP, Dowda M, Pate RR. Perceptions of Physical and Social
Environment Variables and Self-efficacy as Correlates of Self-reported Physical Activity among Adolescent Girls. J Pediatr Psychol. 2007;32(1):6-12.

32. Saunders R, Pate R, Felton G, Dowda M. Development of Questionnaires to Measure Psychosocial Influences on Children's Physical Activity. Prev Med. 1997;26:241-7.

33. Godin G, Shephard R. Godin Leisure-Time Exercise Questionnaire. Med Sci Sports Exerc. 1997:29(6):S36-8.

34. Trost S, Saunders R, Ward D. Determinants of Physical Activity in Middle Schoolchildren. Am J Health Behav. 2002;26(2):95-102.

35. Thilo K, Matthew K, Pei-Shu H, Groah S. The SCI Exercise Self-efficacy Scale (ESES): Development and Psychometric Properties. Int J Behav Nutr Physic Act. 2007;4:34.

36. Bandura A. Exercise Self-efficacy Scale. Autoeficacia para el Entrenamiento Físico. Hallado en: http://www.emory.edu/EDUCATION/ $\mathrm{mfp} /$ self-efficacy.html. Acceso el 15 de septiembre de 2009.

37. Resnick B, Jenkins L. Testing the Reliability and Validity of the Self-efficacy for Exercise Scale. Nurs Res. 2000;49(3):154-9.

38. Luszczynska A, Gutiérrez-Doña B, Schwarzer R. General Self-efficacy in Various Domains of Human Functioning: Evidence from five Countries. Int J Psychol. 2005;40:80-9.

Manuscrito recibido el 13 de mayo de 2008. Aceptado para publicación, tras revisión, el 6 de diciembre de 2008.

ABSTRACT Objectives. To design a questionnaire for assessment of self-efficacy toward physical activity in school children, as well as to measure its construct validity, test-retest reliability, and internal consistency.

\section{New questionnaire to assess self-efficacy toward physical activity in children}

Methods. A four-stage multimethod approach was used: (1) bibliographic research followed by exploratory study and the formulation of questions and responses based on a dichotomous scale of 14 items; (2) validation of the content by a panel of experts; (3) application of the preliminary version of the questionnaire to a sample of 900 school-aged children in Mexico City; and (4) determination of the construct validity, test-retest reliability, and internal consistency (Cronbach's alpha).

Results. Three factors were identified that explain $64.15 \%$ of the variance: the search for positive alternatives to physical activity, ability to deal with possible barriers to exercising, and expectations of skill or competence. The model was validated using the goodness of fit, and the result of $65 \%$ less than 0.05 indicated that the estimated factor model fit the data. Cronbach's consistency alpha was 0.733 ; test-retest reliability was 0.867 .

Conclusions. The scale designed has adequate reliability and validity. These results are a good indicator of self-efficacy toward physical activity in school children, which is important when developing programs intended to promote such behavior in this age group.

Key words Motor activity; self efficacy; reproducibility of results; questionnaires; exercise; child health; Mexico. 